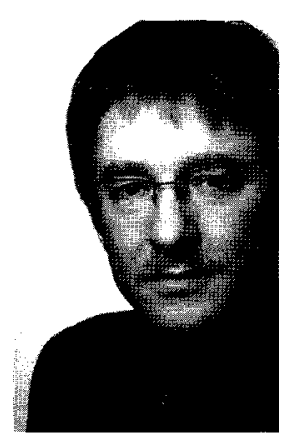

Prof. Dr. Andreas Hänlein، Universität Kassel
Der EuGH hat am 3. April 2008 die TariftreueRegelung des niedersächsischen Landesvergabegesetzes für europarechtswidrig erklärt. Das Urteil (abgedruckt in diesem Heft S. $300 \mathrm{ff}$.) hat viele überrascht. Der Beitrag erläutert die Gründe der Überraschung, analysiert die überzeugende Argumentation des Gerichtshofs und gibt Hinweise zu Auswirkungen des Urteils auf nationaler und auf globaler Ebene.

\title{
Das Rüffert-Urteil des EuGH zum Gebot der „Tariftreue“ bei der Vergabe öffentlicher Aufträge
}

\author{
Andreas Hänlein
}

\section{Einführung}

Tariftreue als Kriterium für die Vergabe öffentlicher Bauaufträge wurde in Deutschland erstmals durch das Berliner Vergabegesetz aus dem Jahr 1999 in gesetzlicher Form vorgeschrieben $^{1} ; 2002$ folgten Tariftreuegesetze in Bayern ${ }^{2}$ und im Saarland ${ }^{3}$. Hintergrund dieser Landesgesetze war die auf das Vergaberechtsänderungsgesetz $1998^{4}$ zurückgehende Regelung des \$97 Abs. 4 GWB. Danach werden öffentliche Aufträge an fachkundige, leistungsfähige und zuverlässige Unternehmen vergeben; abweichend von dieser Regel dürfen andere oder weitergehende Anforderungen an Auftragnehmer nur gestellt werden, wenn dies durch Bundes- oder Landesgesetz vorgesehen ist. Ein Versuch der ersten Regierung Schröder, ein Bundesgesetz zur tariflichen Entlohnung bei öffentlichen Aufträgen zustande zu bringen ${ }^{5}$, scheiterte im Mai 2002 am Bundesrat. Danach entschlossen sich weitere Bundesländer, derartige Regelungen zu erlassen ${ }^{6}$, unter anderem Niedersachsen mit seinem Landesvergabegesetz ${ }^{7}$. Nach dem hier zu besprechenden Urteil der Zweiten Kammer des EuGH vom 3. April 2008

\footnotetext{
1 Berliner Vergabegesetz (VgG Bln) v. 9.7. 1999, GVBl. Bln 1999, S. 369.

2 Bayerisches Bauaufträge-Vergabegesetz (BayBauVG) v. 28.6. 2000, BayGVBl. 2000, S. 364.

3 Saarländisches Bauaufträge-Vergabegesetz (SaarBauVG) v. 23.8.2000, Saar ABl. 2000, S.218.

4 Vergaberechtsänderungsgesetz - VgRÄG v. 26. 8. 1998, BGBl. I, S. 2512.

5 BT-Drs. 14/7796 v. 12.12.2001; kritisch dazu Kämmerer/Thüsing, ZIP 2002, S. $596 \mathrm{ff}$.

6 Aktueller Überblick bei Schulten/Pawicki, WSI Mitteilungen, 2008, S. $184 \mathrm{ff}$. 7 NdsLVG v. 17. 12.2002, GVBl. 2002, S. 370.
}

(Rs. 346/06 Rüffert, abgedruckt in diesem Heft S. 300 ff.) steht nun fest, dass das niedersächsische Landesvergabegesetz mit dem europäischen Recht nicht zu vereinbaren ist. Dieses Urteil ist in der Presse als Überraschung aufgenommen und teilweise heftig kritisiert worden, etwa als „weiterer Schritt zum Raubtierkapitalismus" ${ }^{\text {8 }}$ oder als unglaubliches Urteil, das sich wie eine „Verhöhnung jeglicher Sozialpolitik“ lese?.

Im Folgenden soll in Form einiger Hinweise zum Hintergrund des aktuellen Urteils zunächst gezeigt werden, weshalb die Entscheidung auf den ersten Blick überraschend wirken kann (II). Im Anschluss wird die Argumentation des EuGH analysiert und gewürdigt (III). Einigen Überlegungen zu den Konsequenzen des Urteils für das deutsche Vergaberecht (IV) folgen Hinweise auf seine Bezüge zu Regelwerken des globalen Rechts (WTO und IAO, V).

\section{Zum Hintergrund des Tariftreueurteils}

\section{Der Tariftreuebeschluss des BVerfG}

Aus deutscher Sicht erscheint das Urteil vor allem deshalb überraschend, weil vor nicht allzu langer Zeit, im Jahr 2006, das Bundesverfassungsgericht das Berliner Vergabegesetz in einem umstrittenen Beschluss ${ }^{10}$ als verfassungsgemäß beurteilt

8 So, laut Schlagzeile der Süddeutschen Zeitung v. 4. 4. 2008, S. 1, die IG-Bau.

9 So Prantl im Kommentar der Süddeutschen Zeitung v. 4.4. 2008, S. 4: „Europäische Unsozial-Union“.

10 Polemische Kritik bei Rieble, NZA 2007, S. 1 ff.; kritisch auch Höfling/Rixen, RdA 2007, S. 360 ff.; eingehende Analyse und differenzierende Bewertung bei Preis/Ulber, N.JW 2007, S. $465 \mathrm{ff}$. 
hatte ${ }^{11}$. Das Land Berlin hatte bereits vor Erlass seines Vergabegesetzes bei der Vergabe öffentlicher Bauaufträge Tariftreueerklärungen von den Bietern verlangt, eine Praxis, die das Bundeskartellamt für den Bereich des Straßenbaus 1997 untersagt hatte. Während des Rechtsstreits um diese Untersagungsverfügung trat das Berliner Vergabegesetz in Kraft. Der BGH, der im Rechtsbeschwerdeverfahren mit der Sache befasst war, hielt das Berliner Gesetz für verfassungswidrig und legte es dem BVerfG vor ${ }^{12}$, das - wie gesagt - diese Ansicht nicht teilte. Das Land Berlin sei für den Erlass der betreffenden Regelung zuständig, und diese verstoße auch weder gegen Grundrechte noch gegen sonstiges Bundesrecht. Im Hinblick auf die Koalitionsfreiheit (Art. 9 Abs. $3 \mathrm{GG}$ ) sei, so das BVerfG, bereits der Schutzbereich nicht berührt. Der Schutzgehalt der Berufsfreiheit (Art. 12 Abs. $1 \mathrm{GG}$ ) der betroffenen Unternehmen werde demgegenüber zwar „eingriffsgleich“ beeinträchtigt, da das Gesetz mittelbar Vorgaben mache, wie die Auftragnehmer die Beziehungen zu ihren Arbeitnehmern zu gestalten haben. Dieser Eingriff sei jedoch durch das verfassungsrechtlich legitime Ziel der Bekämpfung der Arbeitslosigkeit im Bausektor in Verbindung mit der Gewährleistung der finanziellen Stabilität des Systems der sozialen Sicherung gerechtfertigt. Zur Erreichung dieses Ziels sei die gesetzliche Tariftreueregelung geeignet, erforderlich und angemessen.

\section{Soziale Zwecke im europäischen Vergaberecht}

Auch aus europäischer Perspektive mag das Urteil überraschend erscheinen, wenn man zunächst einmal die europäische Rechtsentwicklung zur Frage der Zulässigkeit sog. vergabefremder Kriterien bei der Vergabe öffentlicher Aufträge rekapituliert. In einer Reihe von Entscheidungen hat der EuGH entschieden, dass die Berücksichtigung sozialer und umweltbezogener Kriterien bei der Vergabe öffentlicher Aufträge mit den sekundärrechtlichen Regelungen des europäischen Vergaberechts vereinbar sei ${ }^{13}$. So hatte der EuGH in der Rechtssache „Beentjes" die Anforderung gebilligt, bei Ausführung des in Rede stehenden Projekts sollten vom Arbeitsamt vermittelte Langzeitarbeitslose beschäftigt werden, wobei es allerdings nicht zu einer Diskriminierung von Bietern aus anderen Mitgliedstaaten kommen dürfe ${ }^{14}$. Diese Entscheidung wurde vom EuGH in der Rechtssache „Französische Schulen“ im Ergebnis bestätigt, in der es um das gegenüber den Bietern erhobene Ansinnen ging, sich an einem lokalen Pakt zur Beschäftigungsförderung zu beteiligen ${ }^{15}$. In weiteren Entscheidungen billigte der EuGH zudem umweltbezogene Vergabekriterien ${ }^{16}$.

11 BVerfG, Beschluss v. 11.7.2006-1 BvL 4/400-, BVerfGE 116, $202=\mathrm{NJW}$ 2007, S. 51

12 BGH, Beschluss v. 18.1.2000 - KVR 23/98 - NZA 2000, S. $327 \mathrm{ff}$.

13 Eingehende Darstellung bei Wiedmann, Die Zulässigkeit sozialer Vergabekriterien im Lichte des Gemeinschaftsrechts, 2007, S. $39 \mathrm{ff}$.

14 EuGH, Urteil vom 29.9.1988 - Rs. 31/87 - Beentjes -, Slg. 1988, 4635.

15 EuGH, Urteil vom 26.9.2000, Rs. C-225/98 - Kommission ./. Frankreich Französische Schulen -, Slg. 2000, I- 7445; dazu Benedict, NJW 2001, S. $947 \mathrm{ff}$.

16 EuGH, Urteile vom 17.9.2002, Rs. C-513/99 - Concordia Bus -, Slg. 2002 , I- S. 7213, und vom 4. 12.2003, Rs. C- 448/01 - EVN und Winstrom -, Slg. I- 14527 .
Diese Rechtsprechung wurde bei der Reform der Vergaberichtlinien auch vom Gemeinschaftsgesetzgeber aufgegriffen ${ }^{17}$. In den neuen Richtlinien zum Vergaberecht ${ }^{18}$ finden sich nun insbesondere ausdrückliche Regelungen, nach denen die öffentlichen Auftraggeber zusätzliche Bedingungen für die Ausführung ${ }^{19}$ offentlicher Aufträge vorschreiben können, sofern diese mit dem Gemeinschaftsrecht vereinbar sind und in der Bekanntmachung oder in den Verdingungsunterlagen angegeben werden; die zusätzlichen Bedingungen „können insbesondere soziale und umweltbezogene Aspekte betreffen" ${ }^{\text {"20 }}$.

Aus dieser Entwicklung des europäischen Vergaberechts hatte Generalanwalt Bot in seinen Schlussanträgen zur Rechtssache Rüffert den Schluss gezogen, die niedersächsische Tariftreueverpflichtung könne gemeinschaftsrechtlich nicht beanstandet werden ${ }^{21}$.

\section{Bisherige Rechtsprechung des EuGH zum Arbeitnehmerschutz durch Entsenderecht}

Aus europarechtlicher Sicht mag das Urteil des weiteren überraschend erscheinen, wenn man die bisherige Rechtsprechung des EuGH zur Anwendung nationaler arbeitsrechtlicher Schutzvorschriften auf in einen Mitgliedstaat entsandte Arbeitnehmer bedenkt, die für Unternehmen aus anderen EU-Mitgliedstaaten tätig sind.

Nach der ständigen Rechtsprechung des Gerichtshofs verbietet die Dienstleistungsfreiheit (Art. 49 EG) nicht nur jegliche Diskriminierung solcher Unternehmen, sondern gebietet überdies die Aufhebung aller Beschränkungen, die die Tätigkeit im anderen Mitgliedstaat unterbinden, behindern oder weniger attraktiv machen können, sofern eine solche Beschränkung nicht durch zwingende Gründe des Allgemeininteresses gerechtfertigt ist. Die Anwendung arbeitsrechtlicher Schutzvorschriften auf die entsandten Arbeitnehmer ist zwar als eine solche Beschränkung einzuordnen ${ }^{22}$; sie kann allerdings gerechtfertigt sein, denn der Gerichtshof hat den Schutz der (entsandten) Arbeitnehmer als zwingenden Grund des Allgemein-

17 So ausdrücklich jeweils Erwägungsgrund 1 der beiden in der folgenden Fußnote nachgewiesenen RL; vgl. auch Knauff, EuZW 2004, S. 141 ff. und Wiedmann, Die Anhängigkeit sozialer Vergabekriterien im Lichte des Gemeinschaftsrechts, 2007, S. $269 \mathrm{ff}$

18 RL. 2004/17/EG v. 31.3.2004 zur Koordinierung der Zuschlagserteilung durch Auftraggeber im Bereich der Wasser-, Energic- und Verkehrsversorgung sowie der Postdienste, ABl EU v. 30.4.2004 L 134/1; RL 2004/18/EG v. 31.3.2004 über die Koordinierung der Verfahren zur Vergabe öffentlicher Bauaufträge, Lieferaufträge und Dienstleistungsaufträge, ABl EU v. 30.4.2004, L 134/114.

19 Auf die Frage, ob soziale Vergabekriterien nach den neuen Vergaberichtlinien nicht mehr, wie nach der Rechtsprechung des EuGH, bei der Erteilung des Zuschlags, sondern bei der Ausführung des Auftrages zu berücksichtigen sind, soll hier nicht näher eingegangen werden (eingehend hierzu Wiedmann, Die Zulässigkeit sozialer Vergabekriterien im Lichte des Gemeinschaftsrechts, 2007, S. $274 \mathrm{ff}$.).

20 Vgl. etwa Art. 26 RL 2004/18/EG.

21 Schlussanträge des Generalanwalts Yves Bot v. 20.9.2007 zur Rs. C-346/06 Rüffert -, Rz. 132-134; ebenso Bispinck/Kirsch, WSI-Tarifhandbuch 2003, S. 51,69 .

22 Dass sich die Dienstleistungsfreiheit auch auf die Mitnahme von Arbeitnehmern in den Mitgliedstaat erstreckt, in dem der Dienstleistungsauftrag erfüllt werden soll, ist in der Rechtsprechung des EuGH seit langem anerkannt; vgl. EuGH, Urteil v. 27.3.1990, Rs. C-113/89 -, Slg. 1990, I-1439 und EuGH, Urteil v. 9. 8. 1994 - Rs. C-43/93 - Van der Elst -, Slg. 1994, I-3818. 
interesses anerkannt ${ }^{23}$. Gebilligt hat der EuGH im Grundsatz insbesondere auch die Anwendung von Rechtsvorschriften oder Tarifverträgen, die einen Mindestlohn garantieren, auf entsandte Arbeitnehmer eines ausländischen Dienstleisters ${ }^{24}$. Allerdings hat der Gerichtshof auch betont, dass auf diese Weise den betroffenen Arbeitnehmern ein tatsächlicher Vorteil verschafft werden muss, der deutlich zu ihrem sozialen Schutz beiträgt ${ }^{25}$.

In der Literatur ist die These vertreten worden, diese Rechtsprechung könne auf die vergaberechtliche Tariftreueverpflichtung übertragen werden: „Insofern indiziert die Mindestlohnrechtsprechung des EuGH die gemeinschaft(srecht)liche Zulässigkeit der Tariftreueverpflichtung“26. Wer so dachte, wurde nun in der Tat überrascht.

\section{Die Argumentation des Rüffert-Urteils}

Überraschung löste das Urteil des EuGH schließlich auch deshalb aus, weil der Gerichtshof von der Auffassung des Generalanwalts im Ergebnis abgewichen ist ${ }^{27}$. Dies ist allerdings so ungewöhnlich nicht ${ }^{28}$.

\section{Die EntsendeRL als Prüfungsmaßstab}

Hinsichtlich einer Reihe von Aspekten folgt der EuGH dem Generalanwalt freilich zunächst. Seinem Vorschlag ${ }^{29}$ und zugleich dem Vorbringen der Kommission entsprechend wählt der Gerichtshof als Prüfungsmaßstab die Entsenderichtlinie 96/71/ $\mathrm{EG}^{30}$, obwohl sich die Vorlagefrage allein auf die Vereinbarkeit

23 Vgl. insbesondere EuGH, Urteil v. 23. 11. 1999, Rs. C-369 u. 376/96 - Arblade - , Slg. 1999, I -8453, Rz 42, EuGH, Urteil v. 15.3.2001, Rs.C-165/98 - Mazzoleni und ISA -, Slg. 2001, I-2221, Rz 27, EuGH, Urteil v. 24. 1.2002, C-164/99 - Portugaia Construcoes - , Rz 20, und EuGH, Urteil v. 18.7.2002, Rs. C-490/04 - Kommission./.Bundesrepublik Deutschland - , Rz 70, ZESAR 2007, 487 betr. \$2 Abs. 3 AEntG (Anm. Hänlein, ZESAR 2007, 494 ff.).

24 EuGH, Urteil v. 23.11. 1999, Rs. C-369 u. 376/96 - Arblade - , Slg. 1999, I -8453, Rz 42 und EuGH, Urteil v. 15.3.2001, Rs.C-165/98 - Mazzoleni und ISA -, Slg. 2001, I-2221 betr. tariflich festgelegte Mindestlöhne in Belgien; vgl. ferner EuGH, Urteil v. 25. 10.2001, Rs C- 49/98 u. a. - Finalarte -, Slg. 2001, I -7831, 7899, Rz. 31 betr. Urlaubsregelungen in der deutschen Bauwirtschaft, EuGH, Urteil v. 24.1.2002, C-164/99, Slg. 2002, I -787, Rz 21 betr. Mindestlohn in allgemeinverbindlichem Tarifvertrag nach $\$ 1$ Abs. $1 \mathrm{~S} .1 \mathrm{AEntG}$ und EuGH, Urteil v. 12. 10. 2004, C-60/03 - Wolff\&Müller - , Slg. 2004, I- 9553, Rz 36 betr. diesbezügliche Verfahrensmodalitäten (konkret $\$ 1 \mathrm{a} A \mathrm{AEntG}$ ).

25 EuGH, Urteil vom 25. 10.2001, Rs C- 49/98 u. a. - Finalarte -, Slg. 2001, I -7831, Rz 42, und EuGH, Urteil vom 24.1.2002, C-164/99 - Portugaia Construcoes -, Rz. 29.

26 So Wiedmann, Die Zulässigkeit sozialer Vergabekriterien im Lichte des Gemeinschaftsrechts, 2007, S. 253, Klammerzusatz AH, eingehende Begründung in Form einer Verhältnismäßigkeitsprüfung folgt bei Wiedmann, Die Zulässigkeit sozialer Vergabekriterien im Lichte des Gemeinschaftsrechts, 2007, auf S. 254-268; Nachweise zur Gegenmeinung ebd., S. 253, Fn. 1971; vgl. auch Hanau in: Hanau/Steinmeyer/Wank, Handbuch des europäischen Arbeits- und Sozialrechts, 2002, S. 531 (\$ 15 Rz. 493).

27 Schlussanträge des Generalanwalts Yves Bot v. 20.9.2007 zur Rs. C-346/06 Rüffert -.

28 Gerade jüngst war Ähnliches im Vertragsverletzungsverfahren zum AEntG geschehen: vgl. EuGH, 18.7.2007, Rs. C-490/04 - Kommission der Europäischen Gemeinschaften./. Bundesrepublik Deutschland -, ZESAR 2007, 487, und dazu Schlussanträge des Generalanwalts Damaso Ruiz-Jarabo Colomer vom 14.12.2006; vgl. zur betroffenen Sachfrage die Anm. von Hänlein, ZESAR 2007, S. 494, $496 \mathrm{f}$.

29 Schlussanträge des Generalanwalts Yves Bot v. 20.9.2007 zur Rs. C-346/06 Rüffert -, Rz. 57-65.

30 Richtlinie 96/71/EG des Europäischen Parlaments und des Rates vom 16.12. 1996 über die Entsendung von Arbeitnehmern im Rahmen der Erbringung von Dienstleistungen, ABl. 1997, Nr. L 18, S. 1. der niedersächsischen Regelung mit dem Primärrecht bezogen hatte. Im Ausgangsverfahren gehe es um den Einsatz entsandter Arbeitnehmer im Rahmen staatenübergreifender Erbringung von Dienstleistungen, eine Situation, auf die die Entsenderichtlinie nach ihrem Art. 1 Abs. 3 lit. a anzuwenden sei (Rz. 19). Daran könne die vergaberechtliche Einkleidung des Falles nichts ändern (Rz. 20).

Die Wahl der Entsenderichtlinie als Prüfungsmaßstab erweist sich letztlich als die entscheidende Weichenstellung. Dieser Ansatz der rechtlichen Prüfung lässt sich - in Ergänzung der allein auf die Entsenderichtlinie bezogenen Argumentation des Gerichtshofs - auch aus vergaberechtlicher Perspektive überzeugend begründen, denn die neuen Vergaberichtlinien verweisen in ihren Erwägungsgründen ausdrücklich darauf, dass in Situationen grenzüberschreitender Entsendung von Arbeitnehmern die Entsenderichtlinie maßgeblich sein soll ${ }^{31}$.

\section{Richtlinienkonforme Festlegung von Mindestlohnsätzen}

In der Sache heißt es dann im Urteil, die Mitgliedstaaten müssten nach der Richtlinie dafür sorgen, dass entsandten Arbeitnehmern die Einhaltung gewisser Mindestarbeitsbedingungen garantiert werde, u.a. die Einhaltung von Mindestlohnsätzen (Art. 3 Abs. 1 S. 1 lit. c RL 96/71/EG; Rz. 2132). Die Richtlinie spricht, wie der Gerichtshof nun darstellt, unterschiedliche Mechanismen an, mit deren Hilfe die Staaten Mindestarbeitsbedingungen garantieren können. Zunächst verweist die RL auf „Rechts- oder Verwaltungsvorschriften“. Hierzu stellt der Gerichtshof überzeugend fest, das niedersächsische Landesvergabegesetz sei keine derartige Rechtsvorschrift, weil es nicht selbst einen Mindestlohnsatz festlegt (Rz. 24).

Die Richtlinie regelt des weiteren detailliert, unter welchen Voraussetzungen tariflich geregelte Arbeitsbedingungen den Charakter von Mindestarbeitsbedingungen erhalten können. Dabei unterscheidet sie, ob ein Land einen Mechanismus kennt, durch den Tarifverträge für allgemeinverbindlich erklärt werden können, oder ob es dort keinen solchen Mechanismus gibt. Gibt es in einem Land ein derartiges System, wird der Mindeststandard durch den fachlich und räumlich einschlägigen allgemeinverbindlichen Tarifvertrag definiert (Art. 3 Abs. 8 Unterabsatz 1 RL 96/71/EG). Gibt es ein solches System nicht, kann auch auf „normale“ Tarifverträge abgestellt werden, sofern diese in der betreffenden Region für alle vergleichbaren Unternehmen in gleicher Weise gelten und durchgesetzt werden können (Art. 3 Abs. 8 Unterabsätze 2 und 3 RL 96/71/EG) ${ }^{33}$.

31 Vgl. Erwägungsgrund 34 der RL 2004/18/EG: „Für grenzüberschreitende Situationen, in denen Arbeitnehmer eines Mitgliedstaats Dienstleistungen in einem anderen Mitgliedstaat zur Ausführung eines öffentlichen Auftrags erbringen, enthält die Richtlinie 96/71/EG ... die Mindestbedingungen, die im Aufnahmeland in Bezug auf die entsandten Arbeitnehmer einzuhalten sind.“; entsprechend auch Erwägungsgrund 45 der RL 2004/17/EG; zur einschlägigen Entstehungsgeschichte Wiedmann, Die Zulässigkeit sozialer Vergabekriterien im Lichte des Gemeinschaftsrechts, 2007, S. 286.

32 Auch insoweit besteht noch Übereinstimmung mit dem Generalanwalt, vgl. Schlussanträge Rz. 70-75.

33 Die dargestellten Regelungen beziehen sich auf die Bauwirtschaft, vgl. Art. 3 Abs. 1 Unterabsatz 1, 2. Spiegelstrich i. V.m. dem Anhang; allerdings belässt Art. 3 Abs. 10 RL 96/71/EG den Mitgliedsstaaten das Recht, die Anwendung von Arbeits- und Beschäftigungsbedingungen auch dann vorzuschreiben, wenn diese in allgemeinverbindlichen Tarifverträgen geregelt sind, die Tätigkeiten außerhalb der Bauwirtschaft betreffen. 
Im Ausgangsfall ging es darum, den Auftragnehmer und seine Subunternehmer zur Zahlung des „in Tarifverträgen vereinbarten Arbeitsentgelt(s) am Ort der Leistungserbringung“ zu verpflichten. Im erteilten Auftrag wurde dies konkretisiert durch Benennung des in „der Liste der repräsentativen Tarifverträge unter Nr. 01 „Baugewerbe genannten Tarifvertrag(s)“. Dieser Baugewerbe-Tarifvertrag war nicht für allgemeinverbindlich erklärt (Rz. 21, 25, 26 u. 28). Seine Wirkung war im Übrigen auch nicht durch eine Rechtsverordnung nach $\$ 1$ Abs. 3a AEntG auf nicht tarifgebundene Arbeitgeber und Arbeitnehmer erstreckt worden ${ }^{34}$. Da aber das deutsche Arbeitsrecht die Möglichkeit der Erklärung von Tarifverträgen für allgemeinverbindlich kennt, war der Weg versperrt, Mindestarbeitsbedingungen aus schlichten, nicht allgemeinverbindlichen Tarifverträgen abzuleiten (so Rz. $27^{35}$ ). Hilfsweise weist die Kammer darauf hin, dass sich dieses Ergebnis auch bei Anwendung des Unterabsatzes 2 nicht ändern würde, weil das niedersächsische Vergabegesetz den Baugewerbe-Tarifvertrag nicht „allgemein wirksam" im Sinne dieser Vorschrift ${ }^{36}$ mache, sondern die Vergabe privater Aufträge ausspare (Rz. 28 f.).

Das Urteil gelangt damit zu dem solide begründeten Ergebnis, dass der im Baugewerbe-Tarifvertrag vorgesehene Lohn nicht als Mindestlohn im Sinne von Art. 3 Abs. 1 S. 1 lit. c RL anzusehen sei und deshalb dem Auftragnehmer nicht vorgeschrieben werden könne (Rz. $31^{37}$ ).

\section{Die Günstigkeitsklausel}

Der Generalanwalt hatte an dieser Stelle einen argumentativen Weg gesehen, die niedersächsische Regelung gleichwohl als richtlinienkonform einzuordnen: Aus Art. 3 Abs. 7 der RL 96/71/EG sei abzuleiten, dass dem Auftragnehmer die Einhaltung des Bau-Tarifvertrages angesonnen werden dürfe ${ }^{38}$. Diese Regelung versteht die Kammer jedoch grundlegend anders als der Generalanwalt.

Art. 3 Abs. 7 RL 96/71/EG besagt, dass die Garantie von Mindestarbeits- und Beschäftigungsbedingungen durch die Mitgliedstaaten „der Anwendung von für die Arbeitnehmer günstigeren Beschäftigungs- und Arbeitsbedingungen nicht entgegen(steht)“. Diese Klausel lässt sich nun in dreierlei Weise deuten. Zum einen kann man sie verstehen als Vorbehalt zugunsten strengerer Arbeitnehmerschutzvorschriften der Mitgliedstaaten - so, wie etwa Art. 15 Var. 1 der ArbeitszeitRL 2003/88/EG den Mitgliedstaaten erlaubt, „für die Sicherheit und den Gesundheitsschutz der Arbeitnehmer günstigere Rechts- und Verwaltungsvorschriften anzuwenden oder zu erlassen“. Derartige Regelungsvorbehalte zugunsten der Mitgliedstaaten finden ihre primärrechtliche Grundlage in Art. 137

34 Die damals maßgebliche Dritte Verordnung über zwingende Arbeitsbedingun gen im Baugewerbe v. 21.8. 2002, BGB1. I, 2002. S. 3372 bezog sich auf den Tarifvertrag zur Regelung der Mindestlöhne im Baugewerbe im Gebiet der Bundesrepublik Deutschland v. 4.7.2007.

35 Ebenso der Generalanwalt, vgl. Schlußanträge Rz. 86; zu dieser Variante näher EuGH, Urteil v. 18.12.2007 - C.341/05 - Laval, Rz. 65-67.

36 Ausdrückliche Bezugnahme auf Art. 3 Abs. 8 Unterabsatz 21 . Spiegelstrich RL. $96 / 71 / \mathrm{EG}$ in Rz. 28 des Urteils.

37 Vgl. auch die Schlussanträge des Generalanwalts, Rz. 91.

38 Schlussanträge des Generalanwalts, Rz. 93-98.
Abs. 4 EG; danach hindern die auf der Basis dieses Artikels erlassenen Bestimmungen ,die Mitgliedstaaten nicht daran, strengere Schutzmaßnahmen beizubehalten oder zu treffen“. Zweitens könnte man die Klausel im Sinne des herkömmlichen arbeitsrechtlichen Günstigkeitsprinzips auffassen, dass nämlich arbeitsrechtliche Mindeststandards für die Arbeitnehmer günstigere Vereinbarungen durch Einzel- oder Kollektivvertrag nicht ausschließen ${ }^{39}$. Drittens kann man sie nach dem Vorbild des kollisionsrechtlichen Günstigkeitsprinzips deuten, nach dem die Rechtswahl einem Arbeitnehmer nicht den Schutz (günstigerer) zwingender Bestimmungen entziehen darf, den ihm das bei der objektiven Anknüpfung maßgebliche Recht bieten würde (vgl. Art. 30 Abs. $1 \mathrm{EGBGB}^{40}$ ).

Der Generalanwalt hatte die erste Deutungsvariante vertreten. Nach seiner Auffassung handelt es sich bei Art. 3 Abs. 7 RL 96/71/EG um eine Klausel, die ,verstärkten nationalen Schutz" erlaubt ${ }^{41}$. Der EuGH hingegen kombiniert die beiden anderen Deutungsmöglichkeiten: die Klausel erlaube dem Aufnahmemitgliedstaat keine strengeren Schutzvorschriften (Rz. 33) ${ }^{42}$. Das von der EntsendeRL zugelassene Mindestniveau des Arbeitnehmerschutzes kann nach seiner Auslegung vielmehr nur in zwei Fällen überschritten werden (Rz. 34 ${ }^{43}$ ): zum einen kann sich der entsendende Unternehmer im Aufnahmemitgliedstaat aus freien Stücken gegenüber seinem Personal zur Gewährung von Arbeitsbedingungen über dem Mindestniveau verpflichten. Zum anderen kann das richtlinienkonforme Schutzniveau überschritten werden, wenn bereits nach Gesetz oder Tarifvertrag des Herkunftsstaates günstigere Arbeits- und Beschäftigungsbedingungen vorgesehen sind.

Sein Auslegungsergebnis begründet der EuGH zunächst recht lapidar: Die gegenteilige Auslegung, also der Standpunkt des Generalanwalts, nehme der EntsendeRL ihre praktische Wirksamkeit („effet utile“, Rz. 33). Damit ist offenbar gemeint, dass der in der RL niedergelegte Ausgleich zwischen der Dienstleistungsfreiheit der entsendenden Unternehmen - und die Verwirklichung der Dienstleistungsfreiheit ist ausweislich des ersten Erwägungsgrundes das primäre Ziel der EntsendeRL ! - und dem Niveau des Arbeitnehmerschutzes im Zielstaat nicht ausgehebelt werden darf. So gesehen überzeugt die Auslegung des EuGH, geht es doch bei der EntsendeRL gerade nicht um eine auf Art. 137 EG gestützte sozialpolitische Richtlinie mit einem notwendigen Vorbehalt strengerer nationaler Schutzmaßnahmen, sondern um eine Richtlinie zur Koordinierung der Regeln über die grenzüberschreitende Erbringung von Dienstleistungen auf der Grundlage der Artt. 57 Abs. 2 und 66 EGV (Artt. 47 Abs. 2, 55 EG) ${ }^{44}$.

39 Vgl. nur Hanau/Adomeit, Arbeitsrecht, 14. Aufl., 2004, Rz. 50.

40 So Thüsing, Europäisches Arbeitsrecht, 2008, S.262, Rz. 42 („Das Günstigkeitsprinzip macht einen Rechtsvergleich mit den Vorschriften des Herkunftslandes erforderlich") mit S. 331, Rz. 20; zu Art. 30 Abs. 1 EGBGB auch Lakies in Däubler (Hg.), TVG mit AEntG, 2. Aufl. 2006, Anhang 2 zu § 5 TVG, Rz. 20 m. w. N.

41 Schlussanträge des Generalanwalts, Rz. 94.

42 Der EuGH nimmt also eine "Sperrwirkung der Entsenderichtlinie" an; so treffend Kocher, DB 2008, S. 1042, 1043.

43 Erstmals findet sich diese Auslegung in dem vom EuGH zitierten Urteil vom 18.12.2007, Rs. C-341/05 (Laval un Partneri), Rz. 80 f.

44 Vgl. den Einleitungssatz der RL; zum Streit über die Wahl dieser Regelungsgrundlage Thüsing, Europäisches Arbeitsrecht, 2008, S. 254, Rz. 16 m.w. N. 


\section{Tariftreue als ungerechtfertigte Beschränkung der Dienstleistungsfreiheit}

Auf dem Gesichtspunkt der Dienstleistungsfreiheit baut der nun folgende ausführlichere Begründungsansatz des EuGH auf. Er läuft auf eine primärrechtskonforme Auslegung des Art. 3 Abs. 7 der RL hinaus (,im Licht des Art. 49 EG“): Der gesetzliche Zwang zur Zahlung eines tariflichen Entgelts bedeute für den entsendenden Unternehmer eine zusätzliche wirtschaftliche Belastung und behindere ihn bei der der Ausübung der Dienstleistungsfreiheit (Rz. 37). Drei Gesichtspunkte sind es sodann, die der Gerichtshof als potenzielle Gründe zur Rechtfertigung dieser Beschränkung in Betracht zieht, denen er jedoch letztlich allesamt die rechtfertigende Kraft abspricht ${ }^{45}$.

\section{a) Beschränkung der Dienstleistungsfreiheit und Arbeitnehmerschutz}

Der erste potenzielle Rechtfertigungsgrund, mit dem sich der EuGH auseinandersetzt, ist das Ziel des Arbeitnehmerschutzes. Mit diesem Gesichtspunkt hatte er in der Vergangenheit wiederholt Beschränkungen der Dienstleistungsfreiheit gerechtfertigt ${ }^{46}$. Dabei hatte er zugleich klargestellt, dass Beschränkungen der Dienstleistungsfreiheit andererseits nicht durch Ziele wirtschaftlicher Art gerechtfertigt werden könnten, wie etwa den Schutz inländischer Unternehmen ${ }^{47}$. Allerdings war der Gerichtshof höchst zurückhaltend bei der Annahme solcher verbotenen protektionistischen Zielsetzungen: allein auf entsprechende Aussagen in einer Gesetzesbegründung könne die Annahme protektionistischer Absichten nicht gestützt werden, es sei vielmehr eine objektive Betrachtung vorzunehmen und zu untersuchen, ob die jeweilige Regelung tatsächlich zum Schutz der entsandten Arbeitnehmer beiträgt ${ }^{48}$. Hinsichtlich mitgliedstaatlicher Regelungen über Mindestlöhne wie dem deutschen AEntG hat der EuGH darüber hinaus sogar eine Art Vermutung in dem Sinne postuliert, es könne grundsätzlich davon ausgegangen werden, dass der Aufnahmemitgliedstaat mit der Anwendung einer solchen Regelung auf Dienstleistende, die in einem anderen Mitgliedstaat ansässig sind, zum Schutz der Arbeitnehmer handelt ${ }^{49}$.

Vor diesem Hintergrund konnte man der Beschränkung der Dienstleistungsfreiheit also jedenfalls nicht allein wegen der Präambel des niedersächsischen Landesvergabegesetzes die Rechtfertigung absprechen, in der vom Schutz entsandter Arbeitnehmer nicht die Rede ist; sondern davon, dass das Gesetz „Wettbewerbsverzerrungen entgegenwirken (soll), die ...

\footnotetext{
45 Vgl. zur Rechtfertigung von Beschränkungen der Dienstleistungsfreiheit bereits Hänlein, ZESAR 2007, S. 494, 496

46 EuGH, Urteil v. 23.11. 1999, Rs. C-369 u. 376/96 - Arblade -, Slg. 1999 I -8453, 8518, Rz. 51; EuGH, Urteil v. 15.3.2001, Rs. C-165/98 - Mazzoleni und ISA -, Slg. 2001, I-2189, 2222, Rdnr. 26 und EuGH, Urteil v. 25. 10. 2001, Rs C- $49 / 98$ u. a. - Finalarte - Slg. 2001, I -7831, 7899, Rdnr. 33.

47 EuGH, Urteil v. 25. 10.2001, Rs C- 49/98 u. a. - Finalarte -, Slg. 2001, I -7831, Rz 39, und EuGH, Urteil v. 24. 1.2002, C-164/99 - Portugaia Construcoes -, Rz 26.

48 EuGH, Urteil v. 25. 10.2001, Rs C- 49/98 u. a. - Finalarte -, Slg. 2001, I -7831, Rz 40 ff., und EuGH, Urteil v. 24.1.2002, C-164/99 - Portugaia Construcoes -, Rz. 27 f. m. w. N.; skeptisch Junker, JZ 2005, S. 481, 482 f., 488. 49 EuGH, Urteil v. 24.1.2002, C-164/99 - Portugaia Construcoes - , Rz 22, und EuGH, Urteil v. 12.10.2004, C-60/03 - WolfftMüller -, Slg. 2004, I-9553, Rz. 36.
}

durch den Einsatz von Niedriglohnkräften entstehen..... Der Generalanwalt hatte die Meinung vertreten, das niedersächsische Gesetz diene dem Arbeitnehmerschutz, weil es nach Niedersachsen entsandten Arbeitnehmern zu deutlich höheren Löhnen als im Herkunftsstaat verhilft ${ }^{50}$.

Der EuGH ist auch hier anderer Meinung - im Anschluss an die Position der Kommission und Frankreichs ${ }^{51}-$ und nennt dafür zwei Gründe (Rz. 38-40), deren dogmatische Einordnung sich nicht ohne weiteres erschlie $3 \mathrm{t}^{52}$ : Das Erfordernis der Tariftreue sei nicht durch das Ziel des Arbeitnehmerschutzes gerechtfertigt, denn die Einhaltung des tarifvertraglich festgelegten Lohnsatzes werde nur bei einem Teil der Bautätigkeit durchgesetzt, und zwar bei öffentlichen Aufträgen, nicht jedoch bei der privaten Auftragsvergabe. In der Begrifflichkeit einer Verhältnismäßigkeitsprüfung ausgedrückt, spricht der Gerichtshof dem Tariftreuegebot damit die Eignung ab, das Ziel des Arbeitnehmerschutzes zu verwirklichen. Außerdem sei der Tarifvertrag nicht für allgemein verbindlich erklärt worden und gehe im übrigen auch über den Lohnsatz nach ArbeitnehmerEntsendegesetz hinaus ${ }^{53}$. Damit ist wohl gemeint, dass das Tariftreuegebot des niedersächsischen Gesetzes offenbar auch als nicht erforderlich anzusehen ist, weil als milderes Mittel die Vorgabe des allgemeinverbindlichen Mindestlohnes in Betracht käme $^{54}$.

Insgesamt läuft die Argumentation darauf hinaus, dass das proklamierte oder unterstellte Ziel des Arbeitnehmerschutzes nur dann dem Protektionismusvorwurf entgeht, wenn die Arbeitnehmer generell und mit gleicher Wirksamkeit geschützt werden, nicht jedoch nur selektiv. Hatte die bisherige Rechtsprechung großes Verständnis gezeigt für den Zwang zur Anwendung arbeitsrechtlicher Mindeststandards auch auf entsandte Arbeitnehmer, so ist nun eine Grenze markiert, an der sich doch wieder die Dienstleistungsfreiheit durchsetzt.

\section{b) Beschränkung der Dienstleistungsfreiheit und „autonome Ordnung des Arbeitslebens durch Koalitionen"}

Als zweite Möglichkeit einer Rechtfertigung für die Beschränkung der Dienstleistungsfreiheit zieht der EuGH „den Schutz der autonomen Ordnung des Arbeitslebens durch Koalitionen" in Betracht, wobei die Rechtfertigung aus den gleichen Gründen scheitere wie diejenige mit dem Ziel des Arbeitnehmerschutzes (Rz. 41). Dieser Gesichtspunkt ähnelt dem Hinweis des Bundesverfassungsgerichts in seinem Tariftreuebeschluss auf die „Ordnungsfunktion des Tarifvertrages“, die es im Interesse der „autonomen Ordnung des Arbeitslebens durch Koalitionen“ abzustützen gelte, ein Aspekt, den das Bundesverfassungsgericht ergänzend zur Rechtfertigung der Beeinträchtigung der

50 Schlussanträge des Generalanwalts, Rz.118.

51 Vgl. dazu Schlussanträge des Generalanwalts, Rz. 55 f.; im Ergebnis ebenso bereits 2002 Kämmerer/Thüsing, ZIP 2002, S. 596, $598 \mathrm{f}$.

52 Vgl. auch Kocher, DB 2008, S. 1042, 1044: „etwas mysteriöse Begründung“.

53 Das Argument findet sich auch bei Gerken/Löwisch/Rieble, BB 1995, S. 2370, und Thüsing, NJW 2002, S. 2071, 2072; dagegen Wiedmann, Die Zulässigkeit sozialer Vergabekriterien im Lichte des Gemeinschaftsrechts, 2007. S. $260 \mathrm{ff}$.

54 Ebenso die Deutung bei Kocher, DB 2008, S. 1042, 1044. 
unternehmerischen Berufsfreiheit herangezogen hatte ${ }^{55}$. In der Rechtsprechung des EuGH findet sich die Rechtfertigungsfigur in dieser Form bislang, soweit ersichtlich, nicht. Einen ähnlichen Ansatz findet man lediglich in den jüngsten Urteilen des EuGH zur Bewertung gewerkschaftlicher Aktionen gegen grenzüberschreitend tätige Unternehmen ${ }^{56}$. Dies ist auch der Kontext, in dem vermutlich die Rechtfertigung einer Beschränkung der Dienstleistungsfreiheit unter dem besagten Gesichtspunkt am ehesten zu erwarten ist ${ }^{57}$.

\section{c) Beschränkung der Dienstleistungsfreiheit und finanzielle Stabilität der sozialen Sicherungssysteme}

Nicht in dieser Weise neu ist demgegenüber der dritte Gesichtspunkt, den der EuGH als möglichen Rechtfertigungsgrund anspricht (Rz. 42), auf den sich die deutsche Regierung berufen hatte und der auch in der Präambel des niedersächsischen Gesetzes angesprochen ist: der Schutz der finanziellen Stabilität der sozialen Versicherungssysteme ${ }^{58}$. Insoweit fehlten dem Gerichtshof Hinweise in den Akten, dass ohne tariforientierte öffentliche Vergabe eine erhebliche Gefährdung des finanziellen Gleichgewichts des Systems der sozialen Sicherheit drohe; die Rechtfertigung der Beeinträchtigung der Dienstleistungsfreiheit scheiterte insoweit aus tatsächlichen, nicht aus prinzipiellen Gründen - im Gegenteil, der Gerichtshof verweist auf seine Rechtsprechung zur grenzüberschreitenden Inanspruchnahme von Gesundheitsdienstleistungen, in der diese Argumentationsfigur als Rechtfertigungsgrund für Beschränkungen der Dienstleistungsfreiheit etabliert ist ${ }^{59}$. Dass der EuGH diesen Gesichtspunkt jetzt auch im Kontext der Entsendung von Arbeitnehmern so deutlich betont, ist schon eine gewisse Neuigkeit, die nicht zuletzt deshalb bemerkenswert ist, weil auch das BVerfG die „Gewährleistung der finanziellen Stabilität des Systems der sozialen Sicherung“" als ein besonders wichtiges Ziel anerkannt hatte, hinter dem die Freiheit der Unternehmen zur Lohngestaltung zurückzustehen hat ${ }^{60}$. Man könnte sich vorstellen, dass unter diesem Aspekt Beschränkungen der Dienstleistungsfreiheit bei einer nachgewiesenen gravierenden Krise im Bausektor mit entsprechend gravierenden Folgen insbesondere für die Arbeitslosenversicherung gerechtfertigt werden könnten.

55 BVerfG, Beschluss v. 11.7.2006 - 1 BvL 4/400 -, BVerfGE 116, $202=$ NJW 2007, S. 51 sub C II 1 b, bb; diese Argumentationsfigur ist sehr kontrovers diskutiert worden; sehr kritisch: Rieble, NZA 2007, S. 1, 2 (,.Vollends abseitig ..."); freundlicher: Preis/Ulber, NJW 2007, S. 465, 470.

56 Im „Recht auf Durchführung einer kollektiven Maßnahme, die den Schutz der Arbeitnehmer des Aufnahmemitgliedstaats gegen ein etwaiges Sozialdumping zum Ziel hat, "könne ,ein zwingender Grund des Allgemeininteresses im Sinne der Rechtsprechung des Gerichtshofs liegen, der grundsätzlich eine Beschränkung einer der vom Vertrag gewährleisteten Grundfreiheiten zu rechtfertigen vermag“, so EuGH, Urteil v. 18.12.2007, Rs. C- 341/05 - Laval -, Rz. 103; ähnlich auch EuGH, Urteil v. 11. 12.2007, Rs. C-438/05 - Viking -, Rz. 77.

$57 \mathrm{Vgl}$. auch den Hinweis von Kocher, AuR 2008, S. 13 (18 sub 5 c).

58 In der Präambel des niedersächsischen Gesetzes heißt es, das Gesetz „milder(e) Belastungen für die sozialen Sicherungssysteme“.

59 In der unter Rz. 42 zitierten Entscheidung (EuGH, Urteil v. 16. 5. 2006, Rs. C-372/04 - Watts -, Slg. 2006, I- S. 4325 Rz. 103) wird diesbezüglich auf folgende Urteile weiterverwiesen: EuGH, Urteil v. 28.4. 1998, Rs. C-158/96 Kohll -, Slg. 1998, I- S. 1931, Rz. 41, EuGH, Urteil v. 12.7.2001, Rs. C-157/99 Smits und Peerbooms -, Slg. 2001, I- S. 5473 Rz. 72, sowie EuGH v. 13. 5. 2003, Rs. C-385/99, - Müller-Fauré und van Riet, Slg. 2003, I- S. 4509, Rz. 73. 60 BVerfG, Beschluss v. 11.7.2006 - 1 BvL 4/400 -, BVerfGE 116, $202=$ NJW 2007, S. 51 sub C II 2 c, dd (2).

\section{Konsequenzen des Rüffert-Urteils für das deutsche Vergaberecht}

Das Urteil des EuGH in der Rs. Rüffert hat zunächst zur Folge, dass die Regelungen des niedersächsischen Landesvergabegesetzes über die Tariftreueerklärung als unvereinbar mit dem europäischen Recht aufgrund des Vorranges des Gemeinschaftsrechts ${ }^{61}$ gegenüber Auftragnehmern aus anderen EUMitgliedstaaten nicht mehr angewendet werden können. Dies gilt sowohl für bereits erteilte Bauaufträge, wie im Ausgangsfall, wie auch für künftige Ausschreibungen ${ }^{62}$. Auch vergleichbare Regelungen in den Vergabegesetzen anderer Bundesländer können nun nicht mehr angewendet werden ${ }^{63}$. Darüber hinaus wird auch eine Anwendung gegenüber deutschen Bietern kaum mehr möglich sein, denn dies würde auf unterschiedliche Vergabebedingungen in Abhängigkeit von der Staatsangehörigkeit des Bieters hinauslaufen. Auch wenn dies aus europarechtlicher Sicht nicht zu beanstanden sein sollte ${ }^{64}$, würde sich eine Ungleichbehandlung der Bieter in Vergabeverfahren zumindest aus $\$ 97$ Abs. 2 GWB ergeben, wonach die Teilnehmer an einem Vergabeverfahren gleich zu behandeln sind.

Für die künftige Gesetzgebung stellt sich die Frage, in welcher Weise vergabegesetzliche Tariftreueanforderungen ausgestaltet werden könnten. Aus der Argumentation des EuGH zur Entsenderichtlinie lässt sich insoweit ableiten, dass Tariftreueerklärungen, die sich auf die Einhaltung allgemeinverbindlicher Lohntarife beziehen, nicht $z u$ beanstanden wären. Dies gilt außer für Tarifverträge, die nach $\$ 5$ TVG für allgemeinverbindlich erklärt sind, auch für solche, deren Verbindlichkeit durch Rechtsverordnung nach \$1 Abs. 3a AEntG auf nicht tarifgebundene Arbeitgeber und Arbeitnehmer erstreckt ist ${ }^{65}$. Die Durchsetzung der in allgemeinverbindlichen Tarifverträgen der Bauwirtschaft vorgesehenen Lohnsätze mit Mitteln des Vergaberechts wäre als "geeignete Maßnahme“ zur Durchsetzung der EntsendeRL im Sinne von deren Art. 5 anzusehen $^{66}$.

61 Vgl. nur Oppermann, Europarecht, 3. Aufl., 2005, S. 140 und 182 ff.

62 Ebenso Pape, Frankfurter Allgemeine Zeitung, 16.4.2008, S. 23.

63 So auch Last/Dähne in: Deutscher Bundestag, Wissenschaftliche Dienste, Nr. 14/08.

64 Zum Problem der Inländerdiskriminierung etwa Oppermann, Europarecht, 3. Aufl., 2005, S. 524, Rz. 22

65 Dass auch die Erstreckung von Tarifnormen durch Gesetz oder Rechtsverordnung als Allgemeinverbindlicherklärung im Sinne der EntsendeRL anzusehen ist, ergibt sich indirekt aus dem Rüffert-Urteil, denn das niedersächsische Landesvergabegesetz wäre als Instrument der Allgemeinverbindlicherklärung akzeptiert worden, hätte dem nicht seine selektive Wirkung entgegengestanden; ähnlich bereits im Urteil des EuGH v. 23. 11. 1999, Rs. C-369 u. 376/96 Arblade -, Slg. 1999, I -8453, Rz. 46 f.; damals ging es um einen belgischen Tarifvertrag, der durch eine Königliche Verordnung für allgemeinverbindlich erklärt worden war.

66 Fraglich könnte allenfalls sein, ob Landesgesetzgeber etwa durch $\$ 6$ AEntG an der Einführung zusätzlicher vergaberechtlicher Sanktionen zur Durchsetzung allgemein maßgeblicher Standards im Sinne des AEntG gehindert sind. Diese Regelung könnte freilich auch als nicht abschließend aufgefasst werden. 


\section{Bezüge des Rüffert-Urteils zu Regelwerken des globalen Rechts}

Abschließend bleibt darauf aufmerksam zu machen, dass die Problematik der Tariftreue bei der Vergabe öffentlicher Aufträge auch Gegenstand von Regelwerken auf globaler Ebene ist.

\section{Vergaberechtliche Tariftreueanforderungen und das Welthandelsrecht}

Zum einen gibt es ein einschlägiges Regelwerk im Kontext des Welthandelsrechts. Zu bedenken ist hier das „Übereinkommen über das öffentliche Beschaffungswesen" von 1994 (Government Procurement Agreement - GPA), das als plurilaterales Handelsübereinkommen Bestandteil des WTO-Übereinkommens ist. Dieses Übereinkommen haben 39 von 147 Mitgliedern der Welthandelsorganisation ratifiziert, darunter auch die EU mit ihren Mitgliedstaaten ${ }^{67}$. Das Ziel des Übereinkommens besteht in der Öffnung des öffentlichen Beschaffungswesens der Unterzeichnerstaaten für ausländische Anbieter. Ob die Berücksichtigung „vergabefremder Zwecke“ bei der Entscheidung über die Gebote ausländischer Anbieter mit dem Übereinkommen kompatibel ist, ist umstritten und von den Streitbeilegungsgremien der WTO bislang nicht entschieden ${ }^{68}$. Als besonders problematisch wird die Vorgabe angesehen, die steuer-, umwelt- und arbeitsrechtlichen Normen am Auftragsort einzuhalten ${ }^{69}$. In Bezug auf vergaberechtliche Tariftreueanforderungen ist in der Literatur deren Unvereinbarkeit mit dem GPA vertreten worden ${ }^{70}$. Trifft diese Auffassung zu, wäre die Auslegung im Rüffert-Urteil des EuGH zugleich als völkerrechtskonforme Auslegung des Gemeinschaftsrechts einzuordnen. Umgekehrt kann man sich vorstellen, dass die Wertung des EuGH künftige Verfahren vor den WTO Streitbeilegungsgremien beeinflussen könnte.

\section{Vergaberechtliche Tariftreueanforderungen und das Recht der IAO}

Zum anderen ist Tariftreue bei der Vergabe öffentlicher Aufträge ein Thema der Internationalen Arbeitsorganisation. Bereits 1949 hat die Organisation ein Übereinkommen zum Thema verabschiedet, das „Übereinkommen Nr. 94 über die Arbeitsklauseln in den von Behörden abgeschlossenen Verträgen“, ein Übereinkommen, das von 59 Staaten, darunter einige Mitgliedstaaten der Europäischen Union, ratifiziert ist, nicht freilich von Deutschland. Dieses Übereinkommen ist gewissermaßen die Tariftreuekonvention der Internationalen Arbeitsorganisation. Danach müssen Verträge, die gewisse Behörden des jeweiligen Ratifikationsstaats über die Ausführung u.a. von öffentlichen Bauarbeiten schließen, „Klauseln enthalten, die

67 Vgl. dazu Odendahl, EuZW 2004, S. 647, 648.

68 Weiß in Herrmann/Weiß/Ohler, Welthandelsrecht 2. Aufl., 2007, S. 489 f.; allerdings ist eine Änderung des GPA geplant, die die Berücksichtigung vergabefremder Gesichtspunkte ermöglichen soll.

69 Odendahl, EuZW 2004, 647, 652.

70 Bungenberg in: Bauschke et alii (Hrsg.), Pluralität des Rechts - Regulierung im Spannungsfeld der Rechtsebenen, 2002, S. 257, 272. den beteiligten Arbeitnehmern Löhne (einschließlich Zulagen), eine Arbeitszeit und sonstige Arbeitsbedingungen gewährleisten, die nicht weniger günstig sind als die Bedingungen, die im gleichen Gebiet für gleichartige Arbeit in dem betreffenden Beruf oder in der betreffenden Industrie gelten“, und zwar ,auf Grund eines Gesamtarbeitsvertrages oder der Ergebnisse eines anderen anerkannten Verhandlungsverfahrens von Verbänden der Arbeitgeber und der Arbeitnehmer, denen ein wesentlicher Teil der Arbeitgeber und der Arbeitnehmer des betreffenden Berufes oder der betreffenden Industrie angehört, oder (auf Grund) eines Schiedsspruches oder (auf Grund) der innerstaatlichen Gesetzgebung“" (Art. 2). Löhne, Arbeitszeit und sonstige Arbeitsbedingungen der Arbeitnehmer eines Unternehmens, das einen öffentlichen Auftrag im Sinne des Übereinkommens ausführt, müssen sich also an repräsentativen Kollektivverträgen oder an staatlichen Regelungen orientieren. Hinsichtlich der erforderlichen Repräsentativität einer Kollektivregelung ist lediglich erforderlich, dass jeweils „ein wesentlicher Teil der Arbeitgeber und der Arbeitnehmer des betreffenden Berufs oder der betreffenden Industrie“ hinter der Regelung steht, Allgemeinverbindlichkeit wird nicht verlangt.

Auf der diesjährigen Tagung der Internationalen Arbeitskonferenz wird über eine Aktualisierung des Übereinkommens Nr. 94 beraten. Der Sachverständigenausschuss der Organisation hat eine solche Aktualisierung im Grundsatz begrüß $\mathrm{t}^{71}$. Bei den Beratungen sollte das Rüffert-Urteil des EuGH bedacht werden, denn nach diesem Urteil läßt sich ein vergaberechtliches Tariftreueerfordernis auch ohne generell verbindliche Regelung der Arbeitsbedingungen, wie es aus dem Übereinkommen der IAO folgt, nicht ohne weiteres mit dem europäischen Recht vereinbaren.

\section{Fazit}

Der EuGH hat mit seinem Rüffert-Urteil weithin für Überraschung gesorgt. Soweit nationale Tariftreueregelungen sich auf nicht allgemeinverbindliche Tarifverträge beziehen, können diese Regelungen künftig nicht mehr angewendet werden. Die eingangs erwähnte journalistische Kritik (.,Verhöhnung jeglicher Sozialpolitik") erscheint überzogen ${ }^{72}$. Der EuGH hatte in seiner bisherigen Rechtsprechung zum Arbeitnehmerentsenderecht sehr weitgehende Beschränkungen der Dienstleistungsfreiheit nicht immer, aber doch häufig gebilligt und mit teilweise lavierender Argumentation den Vorwurf des Protektionismus zurückgewiesen. Jetzt hat er klargestellt, dass es auch einmal die Dienstleistungsfreiheit sein kann, die sich im Konflikt mit dem jeweiligen nationalen Sozialschutz durchsetzt,

71 Committee of Experts on the Application of Conventions and Recommendations, Labour clauses in public contracts. Integrating the social dimension into procurement policies and practices, International Labour Conference, 97th Session, Report III (Part 1 B), zu finden über

http://www.ilo.org/wcmsp5/groups/public/---ed_norm/---relconf/documents/ meetingdocument/wcms_091400.pdf.

72 Zustimmung zum Ergebnis der Entscheidung auch bei Kocher, DB 2008, S. $1042,1045$. 
und zwar dann, wenn sich der in Rede stehende Sozialschutz letztlich als protektionistisch erweist, weil es nicht darum geht, einen als generell maßgeblich akzeptierten Mindeststandard durchzusetzen. Im Übrigen hat der Gerichtshof sogar weitere argumentative Tore geöffnet, die in Zukunft gegenüber Dienst- leistern aus dem Ausland in Stellung gebracht werden können und deren freiheitsbeschränkendes Potential derzeit nur schwer abzuschätzen ist. Ebenfalls schwer abschätzen lassen sich bislang die Auswirkungen des Rüffert-Urteils auf die Entwicklung globaler Regeln über die Vergabe öffentlicher Aufträge. 